\title{
«POR AHÍ ES UN MARCADOR DISCURSIVO»: UNA PROPUESTA COGNITIVA
}

\author{
María Soledad FunES \\ Universidad de Buenos Aires
}

\section{RESUMEN}

Enmarcado en el Enfoque Cognitivo Prototípico (Langacker 1987, 1991; Lakoff 1987; Hopper 1988, entre otros), que parte del presupuesto de que la Sintaxis está motivada por la Semántica y la Pragmática, y por tanto, emerge del discurso, el presente trabajo analiza la expresión por ahi en un corpus oral de la ciudad de Buenos Aires. Se sostiene la hipótesis de que la expresión por ahi se encuentra en proceso de gramaticalización para ser marcador discursivo, en un camino que va de usos locativos, como en el ejemplo (1) «este último fin de semana fuimos a Puerto Madero porque hacía calorcito y fuimos a pasear por ahi» (PRESEEA, E10: 7), hacia usos modales, como en (2) «el parque Centenario no es un parque tan lindo en términos del parque mismo, tiene por ahí edificios que son lindos como el museo de ciencias naturales y qué sé yo» (PRESEEA, E4: 10). En el camino hacia lo modal resulta de vital importancia la preposición por, que aporta su significado básico locativo, a partir del cual, mediante proyección metafórica, se llega al de modalidad dubitativa. En el uso locativo, por ahí designa un lugar indeterminado, de cierta extensión (en el ejemplo (1), la zona de Puerto Madero). Mediante proyección metafórica, ese lugar indeterminado se vuelve un lugar impreciso en el razonamiento del hablante, quien duda acerca de lo que dice o quiere atenuarlo. La modalidad dubitativa se ve reforzada por otros elementos del contexto (en el ejemplo, la expresión qué sé yo, la negación y la partícula $t a n$ ). Para comprobar la hipótesis, se analizan cualitativa y cuantitativamente ejemplos extraídos del corpus PRESEEA-Buenos Aires, que recoge usos de los años 2010 y 2011.

PALABRAS CLAVE: gramática, preposición, marcador discursivo, metáfora, gramaticalización.

\section{AbSTRACT}

One of the main tenets of the Prototypical-Cognitive Approach is that Syntax is motivated by Semantics and Pragmatics and that it, therefore, emerges from discourse. Guided by this framework, the present study analyzes the expression por ahi recorded in an oral corpus of the city of Buenos Aires. The hypothesis propounded is that the expression is undergoing a grammaticalization process 
to become a discourse marker, on a path that goes from locative usages, as in (1) «este último fin de semana fuimos a Puerto Madero porque hacía calorcito y fuimos a pasear por ahi» («last weekend we went to Puerto Madero because it was warm and went for a walk around there») (PRESEEA, E10: 7) to modal usages, as in (2) «el parque Centenario no es un parque tan lindo en términos del parque mismo, tiene por ahi edificios que son lindos como el museo de ciencias naturales y qué sé yo» («Centenary Park is not such a nice park in itself, what maybe makes it nice is some buildings like the National Museum of Natural Sciences and whatnot») (PRESEEA, E4: 10). On its way towards modality, the preposition por is of overriding importance insofar as it conveys its basic locative meaning from which, through metaphorical projection, it changes to an expression of weak certainty. Regarding locative usage, por ahi denotes an indeterminate place, of certain extension (in example (1), Puerto Madero area). Through metaphorical projection, that indeterminate place becomes a vague location in the speaker's line of reasoning, who, as a result, either doubts about what $\mathrm{s} /$ he says or mitigates it. The weak certainty expression is reinforced by other elements from the context (in the example, the expression qué sé yo/ and whatnot, the negation, and the word $t a n / s u c h)$. In order to test the hypotheses, the examples obtained from the corpus PRESEEA-Buenos Aires, which retrieves usages from 2010 and 2011, are analyzed qualitatively and quantitatively.

KEYWORDS: grammar, preposition, discourse marker, metaphor, grammaticalization.

Fecha de recepción: 11/04/2018

Fecha de aceptación: 31/05/2018

Fecha de la versión definitiva: 11/07/2018

\section{INTRODUCCIÓN}

Respecto de la expresión por ahi, se han postulado diversas hipótesis sobre su significado: Carricaburo (2010) y Di Tullio (2015) reconocen dos formas diferenciadas por un desplazamiento acentual: por ahi, con sentido locativo, y porái, con sentido modal. Para García Negroni y Sauerwein Spinola (2013), por ahi muestra la falta de compromiso del locutor con el punto de vista marcado. Marcovecchio (2015) analiza por ahi como índice de modalidad. Sin embargo, ninguno de los estudios citados realiza un análisis cuantitativo de los datos y en ningún caso se analiza por ahí como marcador discursivo.

En contraposición, en el presente trabajo se realiza un análisis cualitativo y cuantitativo de los usos de por ahi en el corpus oral PRESEEA-Buenos Aires, y se sostiene la hipótesis de que por ahí se encuentra en proceso de gramaticalización para ser marcador discursivo. Ese proceso de gramaticalización se desarrolla desde usos locativos a usos más modales, de imprecisión, atenuación o marca de contraste informativo. En el camino hacia lo modal, la preposición por y el adverbio ahi aportan su significado básico 
locativo, a partir del cual, mediante proyección metafórica, se llega al de modalidad dubitativa, atenuativa o contrastiva. En el uso locativo, por ahi designa un lugar indeterminado, de cierta extensión. Mediante proyección metafórica, ese lugar indeterminado se vuelve un lugar impreciso en el razonamiento del hablante, quien duda acerca de lo que dice o quiere atenuarlo. Este análisis también aporta evidencia sobre el significado básico de por, que sería locativo en lugar de causal, como se ha postulado en varias gramáticas.

\section{BREVE PRESENTACiÓN DEL MARCO TEÓRICO}

El Enfoque Cognitivo-Prototípico (ECP) (cfr. Lakoff 1987; Langacker 1987, 1991; Hopper 1988; Geeraerts 2007, entre otros) sostiene que la gramática no constituye un nivel formal de representación autónomo, sino que se encuentra motivada por la semántica y la pragmática. En consonancia con esta afirmación, el lenguaje no puede separarse tajantemente de otras facultades de la cognición humana, por lo que la intención comunicativa y el punto de vista del hablante resultan fundamentales dentro de la metodología de este enfoque. De esto se desprende que la gramática se caracteriza como una Gramática Emergente del discurso (Hopper 1988). Esto es, las estructuras o regularidades lingüísticas provienen (emergen) de la fijación de rutinas exitosas en el discurso y toman forma a partir de él en un proceso permanente de construcción de la Gramática.

Estos presupuestos teóricos imponen, por tanto, una metodología cualitativa y cuantitativa de análisis de datos auténticos. El hecho de que un hablante elija usar la expresión por ahi para manifestar significados alejados del locativo y cercanos a valores pragmáticos como el dubitativo o el de atenuación se relaciona con el objetivo comunicativo que persigue y con un mensaje específico que quiere transmitir.

\section{SOBRE LA EXPRESIÓN POR AHÍ}

Existen pocos estudios específicos sobre la expresión por ahí. Se destaca, en primer lugar, el de Carricaburo (2010), quien estudia la forma desde la prosodia, estableciendo la variación entre la forma con hiato (por ahí) y la forma diptongada (porái). La autora señala que en la norma culta joven se está produciendo una dicotomía entre la forma con hiato, con valor locativo, y la forma con diptongo como índice de modalidad. La expresión locativa pasa a ser interpretada como un adverbio de duda o de posibilidad, como tal vez o quizás (2010: 371).

Di Tullio y Kornfeld (2013), en un estudio sobre las marcas de modalidad epistémica en el español de la Argentina, señalan que por ahi es una 
marca epistémica de duda o probabilidad, junto con otras formas como capaz y a lo mejor, y lo diferencian de los atenuadores. En un trabajo posterior, Di Tullio (2015) profundiza su análisis de la forma por ahi, reconoce la variación acentual y realiza una asignación similar a la de Carricaburo. El valor locativo de trayecto, lugar por el que alguien o algo se desplaza, se lexicaliza en adverbio modal. La modalidad es epistémica y alude a una actitud de certeza o duda que el hablante asume en relación con el contenido proposicional del enunciado, en términos de su grado de compromiso acerca del valor de verdad, de su ubicación en el mundo real o en uno posible, más o menos alejado del real, o de la evidencia en que se apoya (el testimonio de los sentidos o de lo dicho por otros) (2015: 348).

Desde la Teoría de la Argumentación, García Negroni y Sauerwein Spinola (2013) postulan que la forma por ahi muestra la falta de compromiso del locutor con el punto de vista marcado. Finalmente, Marcovecchio (2015) analiza por ahi como un índice de modalidad, con significado de falta de certeza del hablante frente a sus dichos. A partir de este significado general, Marcovecchio establece una clasificación de distintas interpretaciones de por ahí espacial (es el valor locativo más básico) (Muchos curiosos se habian agolpado en las puertas del teatro Metropolitan Citi para mirar quiénes desfilaban por ahí); aproximación cuantificativa (entre 20 y 26 años, por ahî); lectura evidencial (como lei por ahi); lectura modal y distanciamiento enunciativo del locutor (Lo quiero ya, pero no sé qué va a pasar. Por ahí busca un rival más accesible); interpretación hipotético-probabilística (Creo que todavía estoy construyendo mis personajes. O por ahi los construyo a medias y después los destruyo, y empiezo de nuevo) (2013: 107-111).

Ninguna de las autoras mencionadas analiza por ahi como marcador discursivo, aunque admiten su valor epistémico. Quienes señalan que hubo un proceso de gramaticalización de lo locativo hacia lo modal, no explican de qué modo se dio ese proceso. Tampoco realizan un análisis cuantitativo de los datos que extraen de diferentes corpora.

\section{POR AHÍ COMO MARCADOR DISCURSIVO}

Existe una vastísima producción bibliográfica acerca de los marcadores discursivos $^{1}$. En el presente trabajo, se parte de la definición de marcador

${ }^{1}$ Los marcadores discursivos también fueron denominados enlaces extraoracionales (Gili Gaya 1943), conectores pragmáticos (Briz 1998), enlaces conjuntivos (Fuentes Rodríguez 1987), enlaces textuales (López García 1994), operadores discursivos (Casado 1991), entre otras denominaciones. Resultan fundamentales el estudio inaugural de Schiffrin (1987) y trabajos más actuales como los de Pons Bordería (2008), Santos Río (2003), y Loureda Lamas y Acín-Villa (2010), además del clásico estudio de Martín Zorraquino y Portolés Lázaro (1999). 
discursivo que ofrecen Martín Zorraquino y Portolés Lázaro (1999: 4057): los marcadores discursivos son unidades lingüísticas invariables cuya función es la de guiar las inferencias que se realizan en la comunicación.

En el análisis del corpus, se comprobará que la expresión por ahi cumple con las condiciones postuladas por estos autores (1999: 4059-4080) para ser marcador discursivo: es invariable (por ahí no varía internamente, no se puede cambiar una preposición por otra ni un adverbio por otro sin modificar el significado). No recibe modificadores; tiene distintas posiciones sintácticas: inicial («por ahi últimamente empecé a hacer lo que hace mucho que no hacía que es... eh por ahí ver películas», PRESEEA, E4: pág. 2), intermedia («debe haber alguna peleíta, por ahi, de por medio cada dos por tres», PRESEEA, E4: pág. 12) y final («los bondis son un poco mejor por ahi», PRESEEA, E3: pág. 22); no puede ser negado y afecta a todo el enunciado (no solamente al verbo). Además, presenta un significado que trasciende al conceptual de locativo, que le dio origen, y se acerca a lo que Zorraquino y Portolés denominan significado de procesamiento, es decir, la expresión por ahi otorga una serie de instrucciones semánticas que guía las inferencias que se han de efectuar de los distintos miembros del discurso en los que aparece esta unidad ${ }^{2}$.

Dentro de la clasificación que establecen los autores (1999: 4081), podríamos ubicar a por ahi dentro de los marcadores conversacionales de modalidad epistémica. Los marcadores de este grupo ocurren en géneros dialógicos y señalan el grado de certeza que el hablante atribuye al discurso (1999: 4146). En la lista de ejemplos, aparecen claro, desde luego, por lo visto, pero no aparece mencionado por ahi. Tampoco figura en los diccionarios de Santos Río (2003), Briz, Pons y Portolés (2008) y Fuentes Rodríguez (2009), ni en la Nueva Gramática de la Lengua Española (NGLE) (2009). En la NGLE, en los apartados dedicados a los conectores discursivos, se mencionan aquellos que están formados por preposición + pronombre o adverbio, pero no aparece por ahi. Aparecen las formas con todo, desde luego, por ende, por tanto (2009: $§ 30.121$ ).

Sin embargo, en este trabajo se sostiene la hipótesis de que la expresión por ahi constituye un marcador discursivo que se encuentra en pleno proceso de gramaticalización, y por eso encontramos significados locativos, en el que podemos realizar un análisis interno de la expresión, y otros signi-

${ }^{2}$ Con respecto al requisito de que los marcadores discursivos se encuentren limitados como incisos por la entonación, cabe aclarar que al ser un cuerpo de datos transcripto, no se pudo realizar el análisis prosódico para constatar sus marcas de entonación. Las comas de la transcripción pueden no reflejar fielmente la pronunciación. Queda pendiente, entonces, un estudio fonético prosódico para completar la descripción de por ahí. Sin embargo, el hecho de que la expresión tenga un significado global que no puede dividirse internamente en un análisis sintáctico es prueba de que actúa con cierta autonomía. 
ficados epistémicos, con valores de duda, de atenuación y de contraste, en los que ya no se puede hacer un análisis interno de la forma.

El proceso de gramaticalización se entiende como el proceso donde el material léxico en contextos pragmáticos y morfosintácticos altamente restrictivos se convierte en gramatical (Traugott 1995). El cambio semántico se da de lo concreto a lo abstracto. En este sentido, se propone que en el caso de por ahi, ese pasaje de lo concreto (locativo) a lo más abstracto (epistémico) se desarrolla mediante una proyección metafórica (en términos de Lakoff y Johnson 1980 y 1999) ${ }^{3}$.

En el camino de la metáfora desde lo locativo hacia lo modal resultan de vital importancia tanto la preposición por, que aporta su significado básico locativo de trayecto, como el adverbio ahi, que aporta su significado de lugar indeterminado. En el uso locativo, por ahi designa un lugar indeterminado, de cierta extensión. Mediante proyección metafórica, ese lugar indeterminado se vuelve un lugar impreciso en el razonamiento del hablante, quien duda acerca de lo que dice, quiere atenuarlo o quiere contrastar información. Como veremos en el análisis de los ejemplos, muchas veces la imprecisión acerca de lo dicho se relaciona con la intención deliberada de atenuar las afirmaciones. Se entiende la atenuación en el sentido en que la define Briz (1995, 2007), como una categoría pragmalingüística cuya función es minimizar la fuerza ilocutiva de los actos de habla y el papel de los participantes de la enunciación con el fin de lograr la meta prevista, el acuerdo (o en aminorar el desacuerdo) (1995: 118). Briz señala que la atenuación se manifiesta a través de numerosos recursos lingüísticos, a saber: diminutivos, cuantificadores o partículas, perífrasis, lítote, tiempo condicional, modalidad interrogativa, modalidad de posibilidad, expresiones de ignorancia como no lo sé (se finge ignorancia o incompetencia ante lo dicho por otro interlocutor), y elipsis de la conclusión (2007: 7). Veremos que estas estrategias aparecen con alta frecuencia acompañando a por ahi en los ejemplos que analizaremos.

Briz advierte que la atenuación aparece sobre todo cuando el discurso es polémico y existe desacuerdo entre los interlocutores, y también en el género de la entrevista, ya que es un discurso cuya situación favorece la casi ausencia de actos amenazadores y los que hay siempre están minimizados a través de los atenuantes (2007: 31). Todo esto fue observado en el corpus bajo análisis, dado que el corpus consiste en un conjunto de entrevistas. Ante las preguntas incómodas o que exigen una toma de posición,

\footnotetext{
${ }^{3}$ Para estos autores, la metáfora es entendida como un fenómeno de cognición en el que un dominio se representa conceptualmente en términos de otro. La metáfora se entiende como la proyección de unos conceptos desde un dominio conceptual (el dominio origen) hacia otro dominio conceptual (el dominio destino) (Lakoff y Johnson 1980).
} 
los entrevistados apelan a los recursos de atenuación señalados por Briz, y agregamos, al uso de la expresión por ahí.

\section{El CORPUS}

Los ejemplos analizados se extrajeron del cuerpo de datos oral PRESEEABuenos Aires (Proyecto para el Estudio Sociolingüístico del Español de España y de América), que recoge usos de los años 2010-2011 .

Del total de 90 entrevistas del PRESEEA-Buenos Aires, se seleccionaron 12 entrevistas, que pertenecen al habla culta de la ciudad, debido a que el resto está en proceso de desgrabación. La conversación con cada informante tuvo una duración promedio de 45 minutos y versó sobre diversos temas, como la vivienda, la familia, trabajo, tiempo de ocio, y opiniones sobre tópicos polémicos (como el cambio climático y la despenalización del consumo de drogas).

\section{ANÁlisis DE LOS DATOS}

Se realizó un análisis cuantitativo y cualitativo de los ejemplos de por ahi encontrados en el PRESEEA-Buenos Aires. En el corpus, hay un total de 14 usos de por ahi con significado locativo y un total de 72 ejemplos de por ahi con valor epistémico ${ }^{5}$.

A continuación, un ejemplo con valor locativo ${ }^{6}$ :

(a) E: -¿Y cuál era tu barrio cuando eras chico?

I: -Las Cañitas.

E: ¿¿Dónde es eso, en Capital?

I: -Las Cañitas es eh..por el Hospital Militar, por donde...

E: -¿Donde están los restaurantes ahora?

I: -Claro, por ahí, ahí vive mi vieja, mi vieja vive en la casa misma, en la misma casa de hace mil años. (PRESEEA, E2: pág. 5)

${ }^{4}$ Para más información, véase <http://preseea.linguas.net/>.

${ }^{5}$ En todos los casos, se trata de la variante con hiato, acentuada. En contraposición con lo que señalaban Carricaburo (2010) y Di Tullio (2015), encontramos casos de por ahi acentuado que responden al significado epistémico, además de al significado locativo. No habría, entonces, una distribución complementaria (por ahi asociado al significado locativo y porái asociado al significado modal), sino que una misma forma puede presentar ambos significados dependiendo del contexto.

${ }^{6}$ En la presentación de los ejemplos, en la cita, la «E.» corresponde a «Entrevista», y dentro de cada caso, se distingue con «I» al Informante y con «E» al Entrevistador. Se destaca en cursiva el marcador por ahi, y en algunos casos se subrayan los elementos pertinentes para el análisis. 
En los casos en los que por ahi tiene significado locativo, podemos realizar un análisis sintáctico interno de la expresión. En (a), por ahi es el circunstancial de lugar (preposición y término) que denota una zona indeterminada de Las Cañitas. En contraposición, cuando la expresión por ahi tiene significado modal, no puede hacerse un análisis interno, ya que el significado lo aporta la expresión completa, sin posibilidad de separar las partes.

En el camino de la metáfora hacia los valores modales que veremos en el corpus, se encuentra el ejemplo (b), que se ubica en una zona intermedia, entre el significado locativo y el epistémico:

(b) E.: ¿Qué propondrías para acabar con la inseguridad?

I.: Eh... yo creo que tendría que haber un poco más de control policial, [...] y fundamentalmente creo que que es un tema eh... judicial ya, ¿no?, de de qué pasa con alguien que viola o que mata, y eh... qué condena tiene, digamos... si entra por una puerta y sale por la otra, como suele suceder, o si cumple una condena, creo que pasa más que nada por ahí, ¿no? (PRESEEA, E9: pág. 5)

En este caso, el significado locativo de origen de 'pasar por un lugar físico', se ha proyectado metafóricamente: según el entrevistado, el problema de la inseguridad tiene una raíz judicial porque los delincuentes salen de prisión con facilidad (entra por una puerta y sale por la otra), por lo que para solucionarlo se debería reformar ese aspecto. El tema de la inseguridad, entonces, pasa más que nada por ahi, es decir, es un problema que transita por la vía de las deficiencias judiciales. Un camino metafórico que se deberá sortear para llegar a la solución.

Mediante la metáfora conceptual EL RAZONAMIENTO ES UN LUGAR, que implica que la actividad mental de razonar, de argumentar, se extiende a lo largo de un espacio que se puede transitar, la expresión por ahi llegó a significar valores relacionados con la modalidad dubitativa, la atenuación y el contraste informativo en el camino de la argumentación.

En el análisis cuantitativo de por ahi con valor epistémico se tomaron los siguientes parámetros, surgidos de las interpretaciones semántico-pragmáticas de cada ejemplo: posición, modo verbal, ausencia o presencia de contraste en la argumentación, presencia o ausencia de imprecisión o duda sobre lo dicho, presencia o ausencia de atenuación, presencia o ausencia de expresiones de ignorancia (no sé y qué sé yo), y finalmente, presencia o ausencia de tiempo condicional en el contexto discursivo. En la Tabla 1 vemos los resultados.

De acuerdo con los parámetros medidos, hay una tendencia a que la expresión aparezca en posición intermedia (49 casos, 68,05\%), en modo indicativo ( 70 casos, 97,22\%). Hay una leve tendencia a que la expresión 
TABLa 1. Análisis cuantitativo de por ahí con valores modales en el PRESEEA-Buenos Aires

\begin{tabular}{|c|c|c|}
\hline \multicolumn{2}{|c|}{ Parámetros } & \multirow{2}{*}{$\begin{array}{c}\text { Cantidad de ejemplos } \\
14(19,44 \%)\end{array}$} \\
\hline \multirow{3}{*}{ Posición } & Inicial & \\
\hline & Intermedia & $49(68,05 \%)$ \\
\hline & Final & $9(12,50 \%)$ \\
\hline \multirow{3}{*}{ Modo verbal } & Indicativo & $70(97,22 \%)$ \\
\hline & Subjuntivo & $1(1,39 \%)$ \\
\hline & Sin verbo & $1(1,39 \%)$ \\
\hline \multirow{2}{*}{ Contraste } & Presencia de contraste & $42(58,33 \%)$ \\
\hline & Sin contraste & $30(41,67 \%)$ \\
\hline \multirow{2}{*}{ Imprecisión o duda } & Presencia de duda & $42(58,33 \%)$ \\
\hline & Ausencia de duda & $30(41,67 \%)$ \\
\hline \multirow{2}{*}{ Atenuación } & Con atenuación & $48(66,67 \%)$ \\
\hline & Sin atenuación & $24(33,33 \%)$ \\
\hline \multirow{4}{*}{ Expresiones de ignorancia } & No sé & $17(23,61 \%)$ \\
\hline & Qué sé yo & $15(20,83 \%)$ \\
\hline & Total & $32(44,44 \%)$ \\
\hline & Ausencia & $40(55,55 \%)$ \\
\hline \multirow{2}{*}{ Tiempo condicional } & Presencia & $8(11,11 \%)$ \\
\hline & Ausencia & $64(88,89 \%)$ \\
\hline \multicolumn{2}{|c|}{ Total de ejemplos } & 72 \\
\hline
\end{tabular}

acompañe discursos en los que hay contraste informativo (42 casos, 58,33\%) e imprecisión o duda sobre lo dicho (también 42 casos, 58,33\%). Asimismo, se observa una leve tendencia a la presencia de atenuación (48 casos, $66,67 \%$ ). Además, hay un 44,44\% de presencia de expresiones de ignorancia que acompañan los discursos con por ahi (32 casos en total, 17 para la expresión no sé, y 15 para la expresión qué sé yo). Finalmente, se observan unos pocos casos de tiempo condicional en el contexto lingüístico de por ahi (8 casos, $11,11 \%)$.

A continuación, se analiza cualitativamente una selección de ejemplos a partir del agrupamiento de los usos de por ahi en tres grandes contextos: el de imprecisión o duda, el de atenuación y el de contraste. 


\subsection{La expresión por ahí en contexto de imprecisión o duda}

Cuando la expresión por ahi aparece en contextos de modalidad dubitativa, hablamos de significado de imprecisión o duda. Esta idea también se relaciona con lo que Fuentes Rodríguez (2008) denomina aproximación discursiva. Para esta autora, el concepto de aproximación se encuentra vinculado con la manera en la que el hablante organiza su enunciación de cara al receptor, apuntando a la mostración de la referencia. Se basa en la necesidad del hablante de transmitir una idea, acercando el oyente a la referencia, pero sin llegar a precisarla. Esto ocurre en un ejemplo como el de (1):

(1) E: -¿En general tenés situaciones en las cuales sale el trato de usted o no?

I: - No, muy pocas, muy pocas tirando a cada vez menos, esteeee, estoy pensando si en terapia, pero no tampoco.. eh..

E: -Vos decís de tratar vos de usted a alguien o al revés?

I: -Sí, a veces, por ahi en situaciones como de tránsito o en colectivo qué se yo, pero siempre no superan las dos líneas en ninguna ocasión, así que ...

E: -¿Hay alguna incomodidad que tengas con el tema de usted o..?

I: -Ehh no, me parece que es una cuestión medio generacional a esta altura que salvo en algunas situaciones dee digamos de estar como enfrentado a alguien de otra jerarquía en una cuestión laboral y qué sé yo. (PRESEEA, E4: pág. 1)

En (1), el hablante no logra recordar una situación, piensa, duda, hasta que recupera los viajes en colectivo, pero las califica de pocas (a veces), no recuerda, no sabe (qué sé yo), y son breves (no superan las dos líneas). El marcador por ahi ocurre para introducir las escasas y dudosas situaciones en las que el informante usa el tratamiento de usted. Por ahi expresa la posibilidad en la duda. Los fragmentos subrayados son elementos contextuales que contribuyen a la interpretación dubitativa e imprecisa del discurso. Se trata de cuantificadores y expresiones de ignorancia, como la frase qué sé yo, que aparece dos veces. También hay que considerar los puntos suspensivos, que reflejan una figura tonal sostenida, propia de la modalidad dubitativa.

En (2) vemos un caso en el que no hay tal imprecisión informativa:

(2) E: Bien ¿y para vos qué es un amigo? ¿Qué debería ser o qué, cómo debería ser para ser considerado un buen amigo?

I: Eh, es una frase por ahí trillada cuando uno dice un amigo está en las buenas, en las malas. (PRESEEA, E8: pág. 7)

En este caso, el uso de por ahi se sostiene para salvar la imagen de la entrevistada, para atenuar la adjetivación, anticiparse a lo que puede decir el entrevistador acerca de lo trillado de la frase. Es decir, no habría impre- 
cisión por parte del hablante, sino simplemente atenuación para salvar su imagen por no poder dar una respuesta más original.

\subsection{La expresión por ahí como estrategia de atenuación}

También observamos en el corpus usos de por ahi como estrategia de atenuación. Recordemos que la atenuación se define como una categoría pragmalingüística cuya función es minimizar la fuerza ilocutiva de los actos de habla y el papel de los participantes de la enunciación con el fin de lograr la meta prevista, el acuerdo (o en aminorar el desacuerdo) (Briz 1995: 118).

El fenómeno de atenuación aparece sobre todo ante las preguntas incómodas o que exigen una toma de posición, como sucede en (3):

(3) E: -¿Y cómo considerás que te va en tu profesión, en tu estudio?

I: $\quad-i E s$ la pregunta terrible! Mirá, en el último tiempo por ahí estoy un poco desconforme pero tiene bastante que ver con esto que te decía, que por ahí los últimos.. hasta hace un mes estaba mucho más ocupa$\underline{\text { do, }}$ entonces, este... cerré un par de proyectos de .. de guiones para cine o de guion para animación y qué sé yo. Entonces después me queda un hueco grande y además, como yo hasta el 2008 durante un período de seis años estuve laburando para televisión, estaba como acostumbrado a.. no sé si cierto nivel de ingreso, porque no es por ahi lo que más pesa, pero sí a cierta regularidad con el laburo y ah.. esto mismo que te decía de.. estaba más envalentonado responder: Guionista, este... últimamente siento que no es, no tengo como tan claro hacia dónde va mi carrera ni a qué paso va mi carrera. Entonces ahora si respondo la respuesta va a ser más negativa que otra cosa me parece. (PRESEEA, E4: pág. 4)

La pregunta sobre la profesión logra incomodar al entrevistado, que no sabe cómo decir que no le está yendo bien. Para no decirlo directamente, cuida su imagen dando un circunloquio. El marcador por ahi atenúa junto con el cuantificador un poco el sentido negativo de desconforme. Apela al pasado de trabajo exitoso para no dar cuenta directamente del presente vacío: estaba mucho más ocupado, cerré un par de proyectos. Finge desconocimiento mediante la expresión qué sé yo, hasta que finalmente afirma en presente del indicativo: me queda un hueco grande. La expresión no sé y una nueva ocurrencia de por ahi atenúan el hecho de que le pese no ganar tanto dinero. Finalmente, se confiesa: no tengo tan claro hacia dónde va mi carrera ni a qué paso va mi carrera, y agrega mediante una lítote: si respondo ahora la respuesta va a ser más negativa que otra cosa me parece. Contribuyen también a la interpretación atenuada el uso de la cláusula condicional, el futuro perifrástico y el verbo de creencia me parece. 
En menor medida, en el corpus aparecen ejemplos en los que no hay estrategias de atenuación, como en (4):

(4) E: -Y cuando eras chico, ¿cómo se festejaba la navidad o las pascuas?

I: -Sí, yo creo que más que en casa era... nos reuníamos siempre en casa de los abuelos, mientras estuvieron, o en casa de algún tío, por ahi, que tenía casa más grande. (PRESEEA, E5: pág. 6)

El entrevistado, utilizando un verbo de creencia, dice que las fiestas tenían lugar en la casa de los abuelos, pero abre la posibilidad de la casa del tío con el marcador por ahí. El marcador expresa duda sobre lo dicho, acompañado por el cuantificador algún. No existe la necesidad de atenuar lo dicho, porque ante esa pregunta, el entrevistado no se siente amenazado.

\subsection{Por ahí expresando contraste}

Finalmente, aparece la expresión por ahi en contexto de contraste. Se entiende por contraste lo propuesto por Schwenter (1999: 126), quien sostiene que la operación cognitiva de contraste consiste en la percepción de diferencias en algún nivel y por cualquier medio entre dos entidades o eventos comparables en alguna otra dimensión. A su vez, la expresión de este contraste se apoya lingüísticamente en un fenómeno de diferenciación semántica que se pone de manifiesto a través de términos o sintagmas que se evocan mutuamente con facilidad y que no son necesariamente opuestos léxicos (Schwenter 1999: 127). Así, no habrá solo contraste entre pares como ganar/perder o entre polaridades afirmativas y negativas que acompañan un mismo verbo sino también entre pares del tipo tratar/lograr; creer/ descubrir; a veces/siempre; algunos/muchos; algunos/otros. Además, en consonancia con el enfoque teórico en el que se enmarca el presente trabajo, el contraste es construido por el hablante y reconstruido por el oyente desde la secuencia discursiva en la que los términos conectados aparecen.

En (5), encontramos un ejemplo de por ahi contribuyendo a la expresión de contraste:

(5) E.: ¿Y por qué elegiste esa profesión?

I.: Em... siempre me gustó el inglés, eh... estudié inglés desde muy chica y evidentemente siempre me gustó enseñar aunque no lo tenía del todo claro, de hecho elegí algo que no tiene nada que ver con eso, hasta que me di cuenta que que bueno, que, eh... que por ahi un bioquímico tiene un trabajo mucho más centrado en sí mismo, mucho más, de mucho más aislamiento por ahí que lo que puede ser un un docente que está en contacto con gente todo el tiempo. (PRESEEA, E9: pág. 1) 
Para responder por qué eligió ser profesora de inglés, la entrevistada establece un contraste entre ejercer dicha profesión y haber estudiado Bioquímica. Aparece el marcador por ahi para aventurar la justificación de la elección: que el bioquímico tiene un trabajo centrado en sí mismo. Vuelve a aparecer por ahi, por segunda vez, para marcar el aislamiento y contrastarlo con el trabajo de docente, que está en contacto con gente todo el tiempo.

En el corpus, también hay ejemplos que no presentan contraste, como (6):

(6) E: ¿Y en tu familia cómo se llevan con los vecinos? No te pregunté, vos tenés hijos?

I: tengo hijos, tengo dos, uno que vive afuera y el mayor que vive en Barracas conmigo. Se lleva bien, este no tengo problema, este.

E: Bien, es un trato así, digamos... de vecino.

I: es un trato... me han dicho incluso, alguna vez mi, vecina, mi vecina pegada justo a mi departamento, qué bien, porque en este edificio, como en muchos hay cámara abajo y hay gente que tiene o por ahí justo está mirando la cámara y me han comentado que mi hijo. qué bien que mi hijo no ha dejado pasar a alguien, que le ha preguntado, usted a qué piso va, este, bueno, elogiando la educación o la actitud que había tenido. (PRESEEA, E8: pág. 7)

En (6), la entrevistada no establece ningún contraste para expresar que los vecinos consideran que su hijo está bien educado. El alcance de por ahí es más local, y manifiesta duda sobre lo dicho de sus vecinos.

\section{Conclusiones}

Considerando la interpretación semántico-pragmática de las ocurrencias de por ahi en el corpus PRESEEA-Buenos Aires, concluimos que la expresión por ahi constituye un marcador discursivo, ya que cumple con las condiciones morfosintácticas postuladas por Martín Zorraquino y Portolés Lázaro (1999), a saber: es invariable morfológicamente (no acepta otra preposición u otro adverbio), aparece en distintas posiciones sintácticas (inicial, intermedia y final, con mayor tendencia a aparecer en posición intermedia); no recibe modificadores, no puede ser negado, y presenta un significado que trasciende el conceptual locativo. Se trata de un significado de procesamiento, modal o epistémico, que se relaciona con la modalidad dubitativa, la atenuación y el contraste. En algunos contextos, por ahi se usa en el discurso para dar cuenta de que es impreciso (se duda sobre él o se finge desconocimiento). En otros contextos, por ahi funciona como una estrategia de atenuación para salvar la imagen del hablante ante su interlocutor. Finalmente, en un tercer uso, aparece por ahi en el discurso para establecer contraste informativo. 
Los tres significados encontrados en el corpus responden a una proyección metafórica que ha ocurrido desde el significado locativo de origen. La metáfora consiste en conceptualizar al razonamiento y la habilidad de argumentar del hablante como un lugar extenso en el que las estrategias de argumentación se mueven. Si no se conoce la información, el hablante entonces dudará y su razonamiento se conceptualizará como un lugar indeterminado. Si quiere atenuar sus dichos, su argumentación se mostrará como un lugar no puntual, como una zona de proximidad. Como tercera función discursiva, la argumentación puede tener la finalidad de expresar un contraste entre dos contenidos semánticos con el propósito de responder a una pregunta sin ser directo. En este último sentido, la capacidad de argumentar del hablante se asemeja a un lugar extenso en el que hay dos puntos contrapuestos que hay que mostrar. De este modo, se explica el procedimiento de gramaticalización que está sufriendo la forma por ahí, desde lo locativo a lo modal, en un camino que todavía no ha llegado a su fin. 


\section{BIBLIOGRAFÍA}

BRIZ, Antonio (1995): «La atenuación en la conversación coloquial. Una categoría pragmática». En Luis Cortés (ed.), El español coloquial. Actas del I Simposio sobre Análisis del Discurso Oral, Almería: Servicio de Publicaciones de la Universidad, 103-122.

- (1998): El español coloquial en la conversación. Esbozo de pragmagramática, Barcelona: Ariel.

- (2007): «Para un análisis semántico, pragmático y sociopragmático de la cortesía atenuadora en España y América», Lingüistica Española Actual, 29/1, 5-40.

—, José PorTolés y Salvador Pons (2008): Diccionario de partículas discursivas del español. <http://www.dpde.es>.

CARricaburo, Norma (2010): «La variación acentual en el español de Buenos Aires», Boletín de la Academia Argentina de Letras LXXV, mayo-agosto 2010 (2011), n. ${ }^{\circ} 309-310,363-372$.

Casado Velarde, Manuel (1991): «Los operadores discursivos es decir, esto es, o sea y a saber en español actual: valores de lengua y funciones textuales», Lingüistica Española Actual 13, 87-116.

Di Tullio, Ángela (2015): «Ahi y Por ahi en el español de la Argentina», Anuario de Letras, vol. 1, 2, 327-356.

- y Laura KORNFELD (2013): «Marcas de modalidad epistémica en el registro coloquial». En Ángela Di Tullio (ed.), El español de la Argentina: estudios gramaticales, Buenos Aires: Eudeba, 83-103.

Fuentes Rodríguez, Catalina (1987): Enlaces extraoracionales, Sevilla: Alfar.

- (2008): "Aproximación enunciativa», Lingüistica Española Actual 30, 223-258.

- (2009): Diccionario de conectores y operadores del español, Madrid: Arco/Libros.

García Negroni, María Marta y Sybille Sauerwein Spinola (2013): «Marqueurs de discours et distanciation: une étude contrastive de peut-être, capaz et por ahì, Estudios Románicos 22, 53-64.

GeEraerts, Dirk y Hubert Cuyckens (eds.) (2007): The Oxford Handbook of Cognitive Linguistics, Oxford: Oxford University Press.

GILI GaYA, Samuel (1943): Curso superior de sintaxis española, Barcelona: Biblograf.

Hopper, Paul (1988): «Emergent Grammar and the A Priori Grammar Postulate». En Deborah Tannen (ed.), Linguistics in Context: Connective Observation and Understanding, Ablex: Norwood n. ${ }^{\circ}$ 5, 117-134.

LAKOFF, George (1987): Women, fire and dangerous things, Chicago: University Press.

— y Mark Johnson (1980): Metaphors We Live By, Chicago: Chicago University Press.

— y - (1999): Philosophy in the flesh, Chicago: Chicago University Press.

LANGACKer, Ronald (1987): Foundations of Cognitive Grammar. Theoretical Prerequisites, vol. 1, Stanford: Stanford University Press. 
- (1991): Foundations of Cognitive Grammar. Descriptive Applications, vol. 2, Stanford: Stanford University Press.

LÓPEz GARCíA, Ángel (1994): Gramática del español, I: La oración compuesta, Madrid: Arco/Libros.

LOUREDA LAMAS, Óscar y Esperanza ACín ViLla (coords.) (2010): Los estudios sobre marcadores del discurso en español, Madrid: Arco/Libros.

Marcovecchio, Ana María (2015): «Por ahí/ ni ahi, desde la localización a la modalidad», Spanish in context 12/1, 102-119.

Martín ZorraQuino, María Antonia y José Portolés LÁzAro (1999): «Los marcadores del discurso». En Ignacio Bosque y Violeta Demonte (dirs.), Gramática descriptiva de la lengua española, vol. 3, Madrid: Espasa-Calpe, 4051-4213.

PONS BORDERÍA, Salvador (2008): «Do discourse markers exist? On the treatment of discourse markers in Relevance Theory», Journal of Pragmatics 40, 14111434.

PRESEEA-Buenos Aires (Proyecto para el Estudio Sociolingüístico del Español de España y de América) (2010-2011), Buenos Aires: CONICET.

Real ACAdemia Española (2009): Nueva Gramática de la Lengua Española, Madrid: Espasa.

SAntos Río, Luis (2003): Diccionario de partículas discursivas, Salamanca, LusoEspañola de Ediciones.

Schiffrin, Deborah (1987): Discourse markers, Cambridge: Cambridge University Press.

SCHWENTER, Scott (1999): Pragmatics of conditional marking: Implicature, scalarity and exclusivity, New York: Garland.

TRAUGOTT, Elizabeth (1995): «The role of the development of discourse markers in a theory of grammaticalization». Conferencia presentada en la ICHL XII, Manchester, agosto de 1995. 